\title{
Desenvolvimento e validação de um método cromatográfico em fase gasosa para análise da 3,4-metilenodioximetanfetamina (ecstasy) e outros derivados anfetamínicos em comprimidos
}

\author{
Marcelo Carvalho Lasmar, Edna Maria Alvarez Leite* \\ Setor de Toxicologia, Departamento de Análises Clínicas e Toxicológicas, Faculdade de Farmácia, Universidade \\ Federal de Minas Gerais
}

*Correspondência:

E. M. A. Leite

Faculdade de Farmácia

Departamento de Análises Clínicas e Toxicológicas

Universidade Federal de Minas Gerais Campus da Pampulha

Av. Antônio Carlos 6627 - sala 4136 31270-010 - Belo Horizonte - MG,

Brasil

E-mail: alem@ufmg.br
O uso abusivo das anfetaminas e seus derivados vêm aumentando dramaticamente nos últimos anos em diversas regiões do mundo, notando-se especial utilização do Ecstasy. A análise de amostras da droga apreendidas nas ruas evidenciou, além da presença de MDMA (3,4-metilenodioximetanfetamina), componente principal da droga, outras feniletilaminas, como a MDA (3,4-metilenodioxanfetamina) e MDEA (metilenodioximetiletilanfetamina) este último também conhecido como a droga Eve, ainda pouco difundida no Brasil. O objetivo do presente trabalho foi desenvolver e validar um método analítico confiável, prático e acessivel aos laboratórios de toxicologia, de médio e pequeno porte, no Brasil e em paises em desenvolvimento, para identificação separada do MDMA, MDA e MDEA. A cromatografia em fase gasosa utilizando-se coluna capilar e detector de ionização de chama foi a técnica escolhida. O método analítico apresentou para os três analitos de interesse, faixa ampla de linearidade (1,0 a 500,0 $\mu \mathrm{g} / \mathrm{mL})$; limites de quantificação de $1,0 \mu \mathrm{g} / \mathrm{mL}$ e coeficientes de variação intra e interensaio inferiores a 9,5\%. Os limites de detecção estabelecidos foram 0,7 $\mu \mathrm{g} / \mathrm{mL}, 0,8 \mu \mathrm{g} / \mathrm{mL}$ e $0,6 \mu \mathrm{g} / \mathrm{mL}$, respectivamente para o MDMA, MDA e MDEA. $O$ método foi seletivo na presença de epinefrina, cocaína, anfetamina, ácido acetilsalisílico, metanfetamina, ácido dietilbarbitúrico, paminobenzoil dietilbarbitúrico, paracetamol e cafeina.

\section{INTRODUÇÃO}

O uso abusivo de anfetaminas e seus derivados, que representam a maior classe de estimulantes do sistema nervoso central, vem aumentando drasticamente nos últimos anos em diversas regiões do mundo, especialmente em re- lação à utilização do ecstasy (3,4-metilenodioximetanfetamina) (Cole et al., 2003; Costa, Chasin, 2004; Lapachinske, 2004; Mitrevski et al., 2005; Parrot, 2005; Soares et al., 2004). Segundo dados da Organização das Nações Unidas (ONU), existem, atualmente, 8,3 milhões de usuários de ecstasy no mundo, um aumento de $84,4 \%$ em 
relação aos números divulgados em 2000, que registravam 4,5 milhões de usuários na década passada. A ONU estimou, ainda, que cerca de 1,4 milhões de comprimidos de ecstasy são produzidos anualmente no mundo (média de 168 comprimidos por usuário) sendo os Estados Unidos e a Europa os maiores produtores e consumidores da droga no mundo

A situação é preocupante, também, no Brasil. Em 2004, foram apreendidos 82 mil comprimidos no Rio de Janeiro, quantitativo significativamente superior aos $72 \mathrm{mil}$ comprimidos recolhidos no ano anterior (Monken, 2005). Durante o primeiro semestre de 2003, mais de $55 \mathrm{mil}$ tabletes de ecstasy foram apreendidos em São Paulo (Costa, Chasin, 2004). Em abril de 2005, foram apreendidos 30 mil comprimidos na bagagem de um jovem que desembargava no Brasil, vindo da Holanda (Caruso, Jesus, 2005; Monken, 2005).

O ecstasy é uma droga geralmente utilizada de forma grupal, sendo que seus consumidores são basicamente jovens de classe média-alta e alta (Costa, Chasin, 2004; Lapachinske, 2004; Sardinha, Garcia et al., 2000). Essa droga pode ser administrada de forma isolada ou, na maioria das vezes, associadas com inúmeras outras, como álcool, cocaína, maconha e até benzodiazepínicos (Lapachinske, 2004; Parrot, 2004; Schifano, 2004; Soares et al., 2001).

As análises de amostras de ecstasy apreendidas nas ruas evidência a presença de MDMA (3,4-metilenodioximetanfetamina) e outras feniletilaminas, como MDA (3,4-metilenodioxanfetamina), MDEA (3,4-metilenodioximetiletilanfetamina ou eve), MBDB ( $N$-metilbenzodioxazolilbutamina) e 2C-B (4-bromo-2,5-dimetoxifenilamina), consideradas entactógenos, produzindo sensações de euforia, ânimo e aumento da comunicação, justificando sua crescente popularidade como drogas de raves (Maurer et al., 2000; Soares et al., 2004).

Os efeitos após o uso da droga podem variar consideravelmente, dependendo das características físicas e psicológicas do usuário, da dose, freqüência e duração do uso, e também da variedade de substâncias encontradas nos comprimidos.

O MDMA atua no SNC, ao nível da neurotransmissão serotoninérgica alterando humor, impulsividade, cognição, sono, memória, concentração e implicando em várias doenças neuropsiquiátricas, como depressão e ansiedade, entre outros efeitos (Burguess et al., 2000; Cole, Summall, 2003; Parrot, 2005, 2004; Schifano, 2004). Esse derivado anfetamínico é responsável, também, pela inibição das enzimas monoaminoxidase $\mathrm{A}\left(\mathrm{MAO}_{\triangle}\right)$ e monoaminoxidase $\mathrm{B}\left(\mathrm{MAO}_{\mathrm{B}}\right)$, levando a aumento da concentração sináptica de serotonina, dopamina e norepinefrina. É relatada, também, alteração na neurotransmissão de acetilcolina, histamina e GABA, resultante da afinidade da droga pelos transportadores ou interação direta com os receptores desses neurotransmissores (Cole et al., 2003; Parrot, 2005).

Em administrações agudas o MDMA promove estimulação cardíaca e taquicardia, induzindo uma vasoconstrição. Essas alterações cardiovasculares, associadas à hiperperfusão cerebral podem ser responsáveis pelos inúmeros casos de hemorragias descritas na literatura. (Burguess et al., 2000; Cole et al., 2003; Lapachinske, 2004; Parrot, 2005).

O risco de hepatoxicidade também é descrito na literatura, principalmente quando associado à hipertermia, que pode potencializar a depleção de glutationa induzida pelo MDMA (Burguess, 2000; Cole et al., 2003; Schifano, 2004).

A administração de MDMA produz rápida alteração na função imune, levando a redução no número de linfócitos circulantes, na supressão da proliferação de linfócitos T e na produção de imunoglobulinas, além de alterações na produção de citocinas (Cole et al., 2003).

A presença de outras substâncias nos comprimidos de ecstasy e a ingestão associada a outras drogas como o álcool, pode favorecer o aumento dos efeitos fisiopatológicos do ecstasy (Parrot, 2004; Schifano, 2004).

São, portanto, inúmeros os efeitos descritos resultantes do uso dessa droga, que variam desde as sensações agradáveis (procuradas pelos usuários) até a crises de pânico, psicose e quadros de dependência. (Lapachinske, 2004).

O amplo espectro de efeitos resultante do uso do ecstasy, a falta de estudos científicos sobre o assunto no Brasil, a crescente utilização dessa droga por segmentos da sociedade brasileira, indicam a importância do seu controle.

A análise desses derivados anfetamínicos, em muitos laboratórios de toxicologia forense, é feita por meio de técnicas de imunoensaios, geralmente rápidas e simples, mas que apresentam diversas reações cruzadas, apresentando especificidade inadequada para a identificação das substâncias, sendo, portanto, indicadas apenas para uma triagem toxicológica das drogas. (Talwar et al., 1999). As técnicas cromatográficas são mais confiáveis e específicas para a identificação e quantificação dos derivados anfetamínicos, entre eles o ecstasy. Dentre elas, a mais apropriada é a cromatografia líquida de alta eficiência (CLAE) ou gasosa (CG), utilizando-se detector de espectrometria de massas (Lord et al., 1997; Tagliaro et al., 2000; Talwar et al., 1999; Wood et al., 2005, 2003). É necessário considerar, entretanto, que esse detector eleva o custo da aquisição e da manutenção de um aparelho CLAE ou CG o que, muitas vezes, inviabiliza suas aquisições pela maioria dos labora- 
tórios de pequeno e médio porte, de países em desenvolvimento, como o Brasil.

Assim, esse trabalho objetivou desenvolver e validar um método cromatográfico em fase gasosa com detector de ionização de chama para a análise do MDMA, MDA e MDEA em comprimidos.

\section{MATERIAL E MÉTODOS}

\section{Material}

\section{Equipamentos, Padrões e Vidrarias}

Foi utilizado um cromatógrafo a gás Hewlett Packard ${ }^{\circledR}$ modelo 5890 Series II, equipado com injetor split/splitless, detector de ionização em chama e integrador Hewlett Packard ${ }^{\circledR}$ modelo HP 3395 Integrator.

As substâncias químicas de referência, 3,4-metilenodioxidometanfetamina - MDMA; 3,4-metilenodioxidoanfetamina - MDA; 3,4-metilenodioxidoetilanfetamina - MDEA e $N$-metil-1-(3,4-metilenodioxifenil)-2-butamina) - MBDB, todas grau p.a., utilizadas como padrão foram obtidos da Cerilliant ${ }^{\circledR}$. Soluções metanólicas de diferentes concentrações foram preparadas para o estudo proposto.

Toda vidraria foi descontaminada previamente com solução de hipoclorito de sódio $2 \%$, lavada com água e detergente neutro, para uso em laboratório e enxaguada com água destilada e MiliQ.

\section{Amostras}

Foram utilizados comprimidos clandestinos apreendidos nas ruas pela Polícia Civil de Minas Gerais, suspeitos de conterem ecstasy $(\mathrm{n}=4)$.

Como interferentes na análise foram utilizados comprimidos de anfetamina, metanfetamina, ácido dietilbarbitúrico, $p$-aminobenzoil dietilbarbitúrico, também apreendidos nas ruas pela Polícia Civil; de ácido acetilsalicílico (Aspirina ${ }^{\circledR}$ ), paracetamol $\left(\right.$ Tylenol $^{\circledR}$ ) e paracetamol associado à cafeína $\left(\right.$ Eucodrix $\left.^{\circledR}\right)$ adquiridos no comércio. A solução de epinefrina (Dyfpne-inhal ${ }^{\circledR}$ ) foi obtida em drogaria e o cloridrato de cocaína em pó do Setor de Toxicologia da Faculdade de Farmácia da UFMG.

\section{MÉTODOS}

\section{Condições cromatográficas}

Após otimização foram adotadas as seguintes condições operacionais: coluna cromatográfica capilar Hewlett Packard ${ }^{\circledR}$ modelo HP 5 (5\% de fenil-polimetilsiloxano, $50 \mathrm{~m}$ x 0,32 mm , $0,17 \mu \mathrm{m})$; vazão da fase móvel (He): $2,0 \mathrm{~mL} / \mathrm{min}$; razão de divisão do injetor (split) $1 / 25$, volume injetado de $3 \mu \mathrm{L}$. As temperaturas de operação foram: injetor a $260{ }^{\circ} \mathrm{C}$, detector a $275^{\circ} \mathrm{C}$ e coluna em temperatura programada, iniciando-se em $140^{\circ} \mathrm{C}$ por 2 minutos, seguida de elevação de $5^{\circ} \mathrm{C} / \mathrm{min}$ até $190^{\circ} \mathrm{C}$ e posterior elevação de $35^{\circ} \mathrm{C} / \mathrm{min}$ até $270{ }^{\circ} \mathrm{C}$, permanecendo durante 6 minutos nessa temperatura final.

\section{Preparo da amostra}

Os comprimidos foram triturados e pulverizados separadamente em almofariz e, em seguida, uma alíquota de $10 \mathrm{mg}$ de cada um dos comprimidos foi pesada, transferida quantitativamente para um béquer, adicionada de $10 \mathrm{~mL}$ de metanol e submetida a um banho de ultra-som por 10 min e, em seguida, filtrada sob pressão manual, em filtro RC15, com poros 0,45 $\mu \mathrm{m}$. Dos filtrados obtidos foram transferidos $90 \mu \mathrm{L}$ para um tubo de vidro cônico, adicionado $10 \mu \mathrm{L}$ da solução de MBDB de $500 \mu \mathrm{g} / \mathrm{mL}$ (padrão interno) e injetado $3 \mu \mathrm{L}$ no $\mathrm{CG}$.

\section{Figuras de mérito}

As seguintes figuras de mérito foram validadas no presente trabalho: linearidade, limites de detecção e de quantificação, precisão, especificidade/seletividade e robustez. Essa validação seguiu, basicamente, o exposto por Chasin et al. (1998), em relação às análises toxicológicas.

\section{RESULTADOS E DISCUSSÃO}

\section{Condições cromatográficas}

Os parâmetros cromatográficos obtidos nas condições estabelecidas para o método CG estão mostrados na Tabela I (tempos de retenção médios e de retenção relativos ao padrão interno, fator de retenção e número de pratos teóricos) e na Tabela II (fatores de separação e de resolução).

A Figura 1 mostra um cromatograma referente à análise de soluções contendo MDMA, MDA, MDEA e MBDM (padrão interno) na concentração de $50 \mu \mathrm{g} / \mathrm{mL}$. Pode-se observar a boa resolução e separação dos picos dos analitos de interesse. Verifica-se, também, na Figura 1, a separação adequada entre esses analitos e algumas substâncias que podem estar presentes, como contaminantes, nos comprimidos de ecstasy, mencionadas na Tabela III.

\section{Especificidade ou seletividade}

A especificidade foi considerada como a capacidade do método analítico em detectar o(s) analito(s) de interesse na presença de outros componentes da matriz (Chasin et al., 1998; Ribani et al., 2004). Comprimidos de anfetamina, metanfetamina, ácido dietilbarbitúrico, cloridrato de $p$-aminobenzoil dietilbarbitúrico, parace- 
TABELA I - Tempos de retenção médios, de retenção relativos ao padrão interno, fator de retenção e número de pratos obtidos na análise de MDMA, MDA e MDEA por CG/DIC

\begin{tabular}{lcccc}
\hline Analito & $\begin{array}{c}\text { Tempo Retenção * } \\
\pm \mathrm{DP}(\mathrm{min})\end{array}$ & $\begin{array}{c}\text { Tempo Retenção } \\
\text { Relativo (min)* }\end{array}$ & Fator Retenção & $\begin{array}{c}\mathrm{N}^{\circ} \text { de Pratos } \\
\text { Teóricos }\end{array}$ \\
\hline MDA & $6,83 \pm 0,04$ & 0,82 & 1,79 & 728.889 \\
MDMA & $7,42 \pm 0,02$ & 0,89 & 1,94 & 916.651 \\
MDEA & $7,90 \pm 0,01$ & 0,95 & 2,14 & 1.109 .511 \\
MBDB & $8,35 \pm 0,02$ & 1,00 & 2,31 & 1.422 .908 \\
\hline
\end{tabular}

* média de 10 injeções

TABELA II - Fatores de separação e resolução dos picos cromatográficos obtidos para os compostos de interesse e padrão interno após análise pelo método CG/DIC

\begin{tabular}{lcc}
\hline Analito & $\begin{array}{c}\text { Fator } \\
\text { Separação }(\alpha)\end{array}$ & $\begin{array}{c}\text { Resolução } \\
(\mathrm{Rs})\end{array}$ \\
\hline MDA / MDMA & 1,086 & 36,906 \\
MDMA / MDEA & 1,098 & 30,998 \\
MDEA / MBDB & 1,084 & 32,173 \\
\hline
\end{tabular}

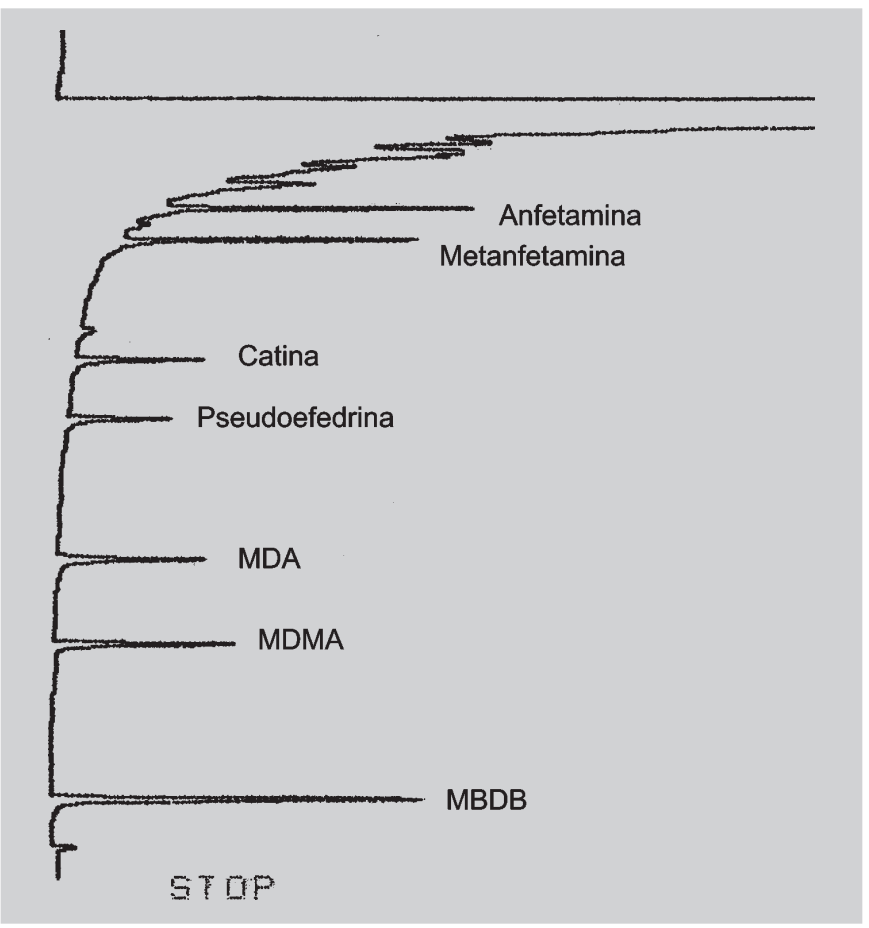

FIGURA 1 - Cromatograma obtido após injeção de solução metanólica contendo MDA, MDMA, MDEA e MBDB (padrão interno), na concentração de $50 \mu \mathrm{g} / \mathrm{mL}$ e algumas substâncias que podem estar presentes nos comprimidos de ecstasy, como contaminantes. tamol, ácido acetilsalicílico e cafeína, além de cloridrato de cocaína (pó) e epinefrina (solução) foram submetidos ao método de análise. Todas as substâncias analisadas apresentaram tempos de retenção distintos daqueles encontrados para os analitos de interesse (Tabela III).

TABELA III - Tempo de retenção e de retenção relativo de alguns dos possíveis interferentes na análise do MDMA, MDA e MDEA por CG, expressos em minutos

\begin{tabular}{lcc}
\hline Analito & $\begin{array}{c}\text { Tempo de } \\
\text { Retenção }\end{array}$ & $\begin{array}{c}\text { Tempo de } \\
\text { Retenção } \\
\text { Relativo }\end{array}$ \\
\hline Epinefrina & 2,42 & 0,29 \\
Cocaína & 3,75 & 0,45 \\
Anfetamina & 4,03 & 0,48 \\
Ácido acetilsalicílico & 4,27 & 0,51 \\
Metanfetamina & 4,61 & 0,55 \\
Ácido dietilbarbitúrico & 6,76 & 0,81 \\
p-Aminobenzoil & 7,03 & 0,84 \\
$\quad$ dietilbarbitúrico & $* *$ & $* *$ \\
Paracetamol & $* *$ & $* *$ \\
Cafeína & 8,35 & 1,00 \\
MBDB (PI) & \\
\hline
\end{tabular}

** sem sinal eletrônico (pico) detectado até 9 minutos após a injeção

\section{Linearidade}

A linearidade é definida como a capacidade do método analítico em fornecer resultados proporcionais à concentração do analito presente na amostra, dentro de uma faixa de concentração, na qual se pode correlacionar o valor de uma variável dependente (medida) por meio de uma variável independente (concentração) (Chasin et al., 1998; Inmetro, 2003).

Soluções padrões de MDMA, MDA e MDEA, em quintuplicata, nas concentrações de 1,$0 ; 2,0 ; 5,0 ; 10,0$; 
50,$0 ; 100,0 ; 250,0$ e $500,0 \mu \mathrm{g} / \mathrm{mL}$, adicionadas de $50,0 \mu \mathrm{g} / \mathrm{mL}$ de MBDB (padrão interno), foram injetadas no cromatógrafo a gás sob as condições analíticas estabelecidas no trabalho.

A resposta do detector apresentou-se linear na faixa de concentração compreendida entre 1,0 a 500,0 $\mu \mathrm{g} / \mathrm{mL}$. As equações das retas e coeficientes de determinação $\left(R^{2}\right)$ encontrados foram:

$\mathrm{y}=0,01334( \pm 0,00013) \mathrm{x}+0,049772( \pm 0,032115)$.

$\mathrm{R}^{2}=0,99844 \pm 0,00093$ para o MDMA;

$\mathrm{Y}=0,01354( \pm 0,00075) \mathrm{x}+0,00108( \pm 0,02161)$.

$\mathrm{R}^{2}=0,00748 \pm 0,00184$ para o MDA e

$\mathrm{Y}=0,02288( \pm 0,00094) \mathrm{x}+0,11174( \pm 0,05044)$.

$\mathrm{R}^{2}=0,99764 \pm 0,00325$ para o MDEA.

\section{Limite de detecção e de quantificação}

O limite de detecção (LD) foi considerado com a menor concentração do analito de interesse presente na amostra, que quando submetido a todo o processo analítico, produz um sinal definido como o limite de detecção do método (Chasin, et al.,1998). No presente trabalho foi estabelecido como LD, a menor concentração dos analitos, detectável pelo procedimento descrito nos itens 2.2.1 e 2.2.2, que apresentou um coeficiente de variação próximo, mas não superior, a 20\%. Nas condições estabelecidas no presente trabalho, esses limites foram $0,7 \mu \mathrm{g} / \mathrm{mL}$ para o MDMA, $0,8 \mu \mathrm{g} / \mathrm{mL}$ para o MDA e $0,6 \mu \mathrm{g} / \mathrm{mL}$ para o MDEA.

O limite de quantificação (LQ) foi estabelecido como sendo a menor concentração dos analitos de interesse que pode ser medida com uma precisão estabelecida (Chasin, et al.,1998); nesse trabalho foi considerado "precisão estabelecida", um coeficiente de variação igual a $10 \%$ o que resultou, para os três analitos de interesse, em um LQ igual a $1,0 \mu \mathrm{g} / \mathrm{mL}$.

\section{Precisão analítica}

A precisão de um método analítico é o parâmetro que avalia a proximidade entre várias medidas efetuadas em uma mesma amostra (Chasin et al., 1998).

Pode ser expressa por meio do coeficiente de variação (CV) ou desvio padrão relativo intra-ensaio (condição de repetibilidade) e interensaio (precisão intermediária).

O CV intra-ensaio foi determinado por meio da análise, em um mesmo dia, de soluções padrões de MDMA, MDA e MDEA, em quintuplicata, nas concentrações de 1,$0 ; 10,0$ e $100 \mu \mathrm{g} / \mathrm{mL}$, adicionadas de $50 \mu \mathrm{g} / \mathrm{mL}$ de MBDB (padrão interno).

$\mathrm{OCV}$ interensaio foi determinado por meio da deter- minação da precisão intermediária uma vez que apenas uma condição analítica, o dia da análise, foi modificada no presente estudo. Para a determinação dessa precisão interensaio, soluções padrões de MDMA, MDA e MDEA, em quintuplicata, nas concentrações de 1,$0 ; 10,0$ e $100,0 \mu \mathrm{g} / \mathrm{mL}$, adicionadas de $50 \mu \mathrm{g} / \mathrm{mL}$ de MBDB (padrão interno) foram analisadas por cinco dias seguidos.

As precisões intra e interensaio foram avaliadas por meio da determinação do coeficiente de variação $(\mathrm{CV})$ das áreas relativas (razão entre área absoluta dos analitos e a área absoluta do padrão interno).

A Tabela IV apresenta os resultados, dos coeficientes de variação intra e interensaio, expressos em porcentagem, estabelecidos para as substâncias.

TABELA IV - Coeficientes de variação intra e interensaio (precisão intermediária) do método CG/DIC para a análise de MDMA, MDA e MDEA

\begin{tabular}{lccc}
\hline & \multicolumn{3}{c}{ Concentrações $(\mu \mathrm{g} / \mathrm{mL})$} \\
& 1,0 & 10,0 & 100,0 \\
\hline Precisão Intra-ensaio & \multicolumn{3}{c}{ CV $(\%)$} \\
MDMA & 9,46 & 4,54 & 1,51 \\
MDA & 9,25 & 2,91 & 0,97 \\
MDEA & 6,24 & 1,62 & 1,07 \\
\hline Precisão Intermediária* & \multicolumn{3}{c}{ CV $(\%)$} \\
MDMA & 7,25 & 4,93 & 3,21 \\
MDA & 7,11 & 3,97 & 3,00 \\
MDEA & 4,10 & 3,00 & 2,30 \\
\hline
\end{tabular}

* 5 dias subseqüentes

\section{Robustez}

A robustez de um método analítico avalia a resistência a variações nos resultados quando pequenas diferenças são realizadas nas condições experimentais inicialmente descritas ou seja, um método é considerado robusto quando seus resultados não são afetados por pequenas modificações em seus parâmetros analíticos (Inmetro, 2003; Iupac, 2002).

Nesse estudo, foram testadas pequenas variações nas condições cromatográficas, como alterações na vazão da fase móvel (de 2,0 $\mathrm{mL} / \mathrm{min}$ para 1,8 e $2,2 \mathrm{~mL} / \mathrm{min}$ ) e na programação da temperatura da coluna cromatográfica tanto nas rampas de incremento de temperatura quanto no tempo de duração das etapas isotérmicas. Essas alterações não causaram diferenças significativas nos parâmetros de identificação e quantificação dos analitos de interesse, revelando a robustez do método utilizado. 


\section{Estabilidade}

A estabilidade química dos compostos MDMA, MDA e MDEA foi determinada utilizando-se soluções metanólicas dos analitos, na concentração de $2,0 \mu \mathrm{g} / \mathrm{mL}$, acondicionadas em frascos de vidro âmbar e mantidas sob congelamento a $-20^{\circ} \mathrm{C}$. Após o preparo das soluções, alíquotas foram analisadas em um mesmo dia, sendo o resultado obtido considerado como sendo o do tempo zero do experimento. Os resultados obtidos nas análises subseqüentes, realizadas após descongelamento em diferentes dias, foram comparados com aquele encontrado no tempo zero, aceitando-se uma variação máxima de 20\%. Nessas condições as substâncias mostraram-se estáveis por, no mínimo, por 27 dias.

\section{Aplicação do método validado às amostras}

O método analítico CG/DIC, após validação, foi aplicado na análise de quatro comprimidos apreendidos pela Polícia Civil de Minas Gerais, cuja caracterização como "derivados de anfetaminas" havia sido realizada no laboratório daquele Órgão, por meio de cromatografia gasosa utilizando-se detector de espectrometria de massas. A quantificação dos compostos de interesse foi realizada com o auxílio de curvas analíticas preparadas com soluções padrões de MDMA, MDA e MDEA, nas concentrações de 5,$0 ; 50,0 ; 100,0 ; 250,0$ e $500,0 \mu \mathrm{g} / \mathrm{mL}$ e de MBDB (padrão interno) na concentração de $50 \mu \mathrm{g} / \mathrm{mL}$, analisadas em triplicatas. A Figura 2 mostra o cromatograma obtido após a análise de um desses comprimidos (comprimido 1), pelo método validado.

Todos os quatro comprimidos analisados apresentaram teores variados de MDMA, mas somente no comprimido 1 foram detectados MDA e MDEA. Esse resultado foi coincidente aos da Polícia Civil, demonstrando a eficácia do método proposto. No caso do comprimido 1, foi encontrada uma concentração de MDMA bastante elevada, acima da faixa de linearidade estudada no presente trabalho. O extrato obtido do comprimido 1 foi diluído 10 vezes com metanol e somente assim, após uma nova injeção no cromatógrafo a gás, foi possível quantificar o MDMA presente na amostra.

Os resultados da análise de cada um dos comprimidos apreendidos, com os respectivos teores das substâncias pesquisas são apresentados na Tabela $\mathrm{V}$.

Pelos dados apresentados pode ser observado que a aplicação do método cromatográfico com detecção por ionização de chama apresentou-se bastante adequada para a identificação de ecstasy e outros derivados de anfetamina em comprimidos clandestinos. Foi possível detectar e quantificar de maneira isolada os três principais derivados anfetamínicos que constituem os comprimidos de ecstasy, o que representa uma grande vantagem em relação a outros métodos analíticos freqüentemente utilizados em laboratórios de toxicologia forense brasileiros, como por exemplo, técnicas de imunensaios.

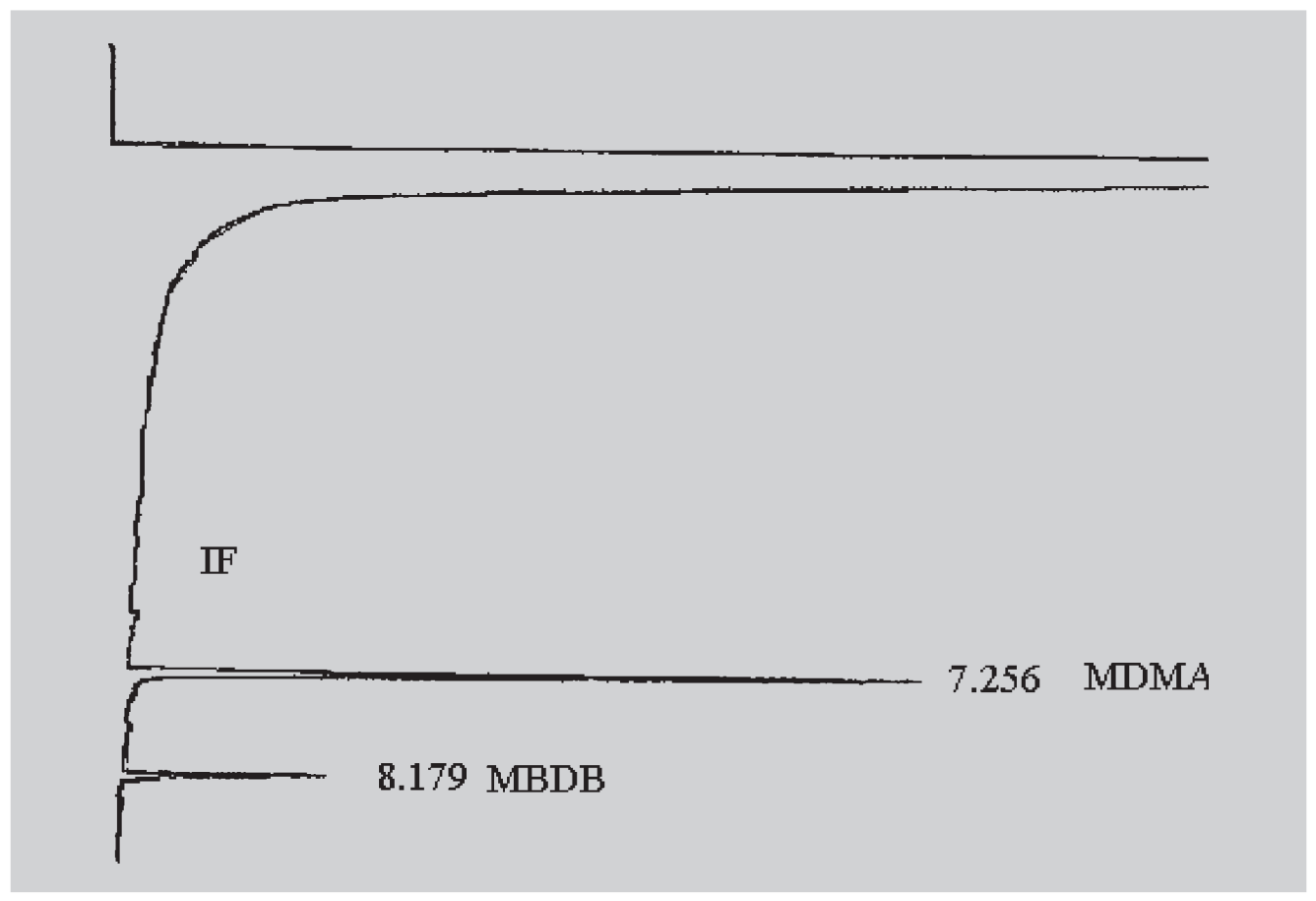

FIGURA 2 - Cromatograma obtido após a análise do comprimido ${ }^{\circ} 01$ de ecstasy pelo método CG/DIC. 
TABELA V - Teores de MDMA, MDA e MDEA encontrados nos comprimidos apreendidos pela Polícia Civil de Minas Gerais e analisados por CG/DIC

\begin{tabular}{lccc}
\hline Comprimido & $\begin{array}{c}\text { MDMA } \\
(\mathrm{mg})\end{array}$ & $\begin{array}{c}\text { MDA } \\
(\mathrm{mg})\end{array}$ & $\begin{array}{c}\text { MDEA } \\
(\mathrm{mg})\end{array}$ \\
\hline 01 & 218,3 & 3,3 & 1,5 \\
02 & 49,1 & - & - \\
03 & 53,3 & - & - \\
04 & 50,0 & - & - \\
\hline
\end{tabular}

A obtenção de picos cromatográficos com resolução apropriada, a faixa de linearidade ampla, que permitiu quantificar com segurança, desde concentrações traços de MDA e MDEA até níveis elevados de MDMA, a seletividade frente a várias possíveis substâncias interferentes e a robustez em condições cromatográficas variadas revela a utilidade e confiabilidade desse método GC/DIC na identificação e quantificação específica dos três derivados anfetamínicos.

Outra vantagem do método é a simplicidade e rapidez da extração dos analitos presentes nos comprimidos. Avalia-se que a precisão analítica encontrada, tanto em ensaios em condições de repetitividade quanto de precisão intermediária, foi bastante satisfatória considerando a faixa de sensibilidade dos detectores de ionização de chama e as baixas concentrações dos produtos que puderam ser quantificadas $(1 \mu \mathrm{g} / \mathrm{mL})$.

\section{CONCLUSÃO}

Os resultados desse trabalho indicam a validade do uso da cromatografia em fase gasosa, utilizando-se detector de ionização de chama (CG/DIC), na identificação dos principais componentes dos comprimidos de ecstasy. Esse método apresenta-se como opção confiável aos laboratórios de toxicologia forense, de porte médio e pequeno, que não dispõem de um detector de massas para a identificação e quantificação de comprimidos de ecstasy.

\section{ABSTRACT \\ Development and validation of a gas chromatography method for determination of ecstasy and amphetamines derivatives in tablets}

The abusive use of the amphetamine derivative ecsyasy in the world come increasing in the last years. Many tablets samples kept on the streets shown the presence not only of the MDMA-3,4-methylenedioxymethamphetamine, the main drug component but also of the MDA-3,4methylenedioxyamphetamine and MDEA - 3,4methylenedioxymethylethylamphetamine. The present study sought to develop and validate an analytical method for determination of MDMA, MDA and MDEA in tablets to be accessible for the most small or medium laboratories of toxicology of the development countries as Brazil. It was chosen for development and validation a gas chromatography method with flame ionization detection. The analytic validation results for MDMA, MDA and $M D E A$ were linearity range of 1.0 to $500.0 \mu \mathrm{g} / \mathrm{mL}$, intra and interassay coefficient of variation lower than $9.5 \%$ and quantification limit of $1.0 \mu \mathrm{g} / \mathrm{mL}$. The detection limits were $0.7 \mu \mathrm{g} / \mathrm{mL}, 0.8 \mu \mathrm{g} / \mathrm{mL}$ and $0.6 \mu \mathrm{g} / \mathrm{mL}$ respectively to MDMA, MDA and MDEA. The method showed a good seletivity as the epinephrine, cocaine, amphetamine, methamphetamine, acethyl salicilic acid, diethyl barbituric acid, p-aminobenzoyl diethyl barbituric, paracetamol and caffeine presences did not interfere with the measurement of the three analytes.

UNITERMS: Ecstasy. Amphetamine derivative. Gas chromatography determination. Flame ionization detection.

\section{REFERÊNCIAS BIBLIOGRÁFICAS}

BURGESS, C.; O’DONOHOE, A.; GILL, M. Agony and ecstasy: a review of MDMA effects and toxicity. Eur. Psychi., v.15, p. 287-294, 2000.

CARUSO, M.; JESUS, D. Anova era do ecstasy. Revista Isto É, São Paulo, no 1853 , p. 62-65. (Caderno Comportamento).

CHASIN, A.M.; CHASIN, M.; SALVADOR, M. C. Validação de métodos cromatográficos em análises toxicológicas. Rev. Farm. Bioquim. Univ. S. Paulo, v.30, n.2, p. 49-53, 2004.

CHASIN, A.M.; NASCIMENTO, E. S.; NETO, L. M. R.; SIQUEIRA, M. E. P. B.; ANDRAUS, M. H.; SALVADOR, M. C.; FERNÍCOLA, N. A.G.; GORNI, R.; SALCEDO, S. Validação de métodos em análises toxicológicas: uma abordagem geral. Rev. Bras. Toxicol., v.11, n.1, p.1-6, 1998.

COLE, J.C.; SUMNALL, H. R. The pre-clinical behavioral pharmacology of 3,4-methylenedioxymethamphetamine (MDMA). Neurosci. Biobeh. Rev., v.27, n. 3,p.199-217, 2003. 
COSTA, J. L.; CHASIN, A. A.M. Determination of MDMA, MDEAA and MDA in urine by high performance liquid chromatography with fluorescence detection. $J$. Chromatog., B, v.811, p. 41-45, 2004.

Instituto Nacional de Metrologia, Normalização e Qualidade Industrial - INMETRO. Orientações sobre validação de métodos de ensaios químicos, DOQ-CGCRE-008, 2003.

International Union of Pure and Applied Chemistry - IUPAC. Analytical, applied, clinical, inorganic and physical chemistry division. Interdivision working party for harmonization of quality assurance schemes for analytical laboratories. Harmonized guidelines for single laboratory validation of method of analysis. Pure Appl. Chem., v.74, n.5, p. 835-855, 2002.

LAPACHINSKE, S. F. Quantificação de amostras de $M D M A$ por cromatografia em fase gasosa (CG/NPD). São Paulo. 2004. 93p. [Dissertação de Mestrado. Faculdade de Ciências Farmacêuticas. Universidade de São Paulo].

MAURER, H. H.; BICKEBOELLER-FRIEDRICH, J.; KRAEMER, T.; PETERS F.T. Toxicokinetics and analytical toxicology of amphetamine-derived designer drugs ('Ecstasy'). Toxicol. Lett., v. 112 -113, p.133-142, 2000.

MITREVSKI, B.; ZDRAVKOVSKI, Z. Rapid and simple method for direct determination of several amphetamines in seized tablets by GC-FID. Forensic Sci. Int., v. 152, n.2-3, p. 199-203, 2005.

MONKEN, M.H. PF apreende 31 mil pílulas de ecstasy. Folha de São Paulo, São Paulo,16 abr., p. C1, 2005. (Caderno Folha Cotidiano).

PARROTT, A.C. Chronic tolerance to recreational MDMA (3,4-methylenedioxymethamphetamine) or Ecstasy. $J$. Psychopharmacol., v 19, n.1, p. 71-83, 2005

PARROTT, A.C. Is ecstasy MDMA? A review of the proportion of ecstasy tablets containing MDMA, their dosage levels, and the changing perceptions of purity. Psychopharmacol., v. 173, n. 3-4, p. 234-241, 2004.

RIBANI, M.; BOTTOLI, C. B. G.; COLLINS, C. H.; JARDIN, I.C.S.F.; MELO, L. F. C.; Validação em métodos cromatográficos e eletroforéticos. Quim. Nova, v. 27, n.5, p.771-180, 2004.
SARDINHA, L.S.; GARCIA, A.L. Um estudo sobre a psicodinâmica do usuário de ecstasy. Psicologia: Teoria e Prática, v. 2, n.2, p.70-87, 2000.

SCHIFANO, F. A bitter pill. Overview of ecstasy (MDMA, MDA) related fatalities. Psychopharmacology, v. 173, n.3-4, p. 242-248, 2004.

SOARES, M. E.; CARVALHO, F.; BASTOS, M. L. Determination of amphetamine and its metabolite $\mathrm{p}$ hydroxyamphetamine in rat urine by reversed-phase highperformance liquid chromatography after dabsyl derivatization. Biomed. Chromatog., v. 15, n.7, p.452456, 2001.

SOARES, M.E.; CARVALHO, M.; CARMO, H.; REMIAO, F.; CARVALHO, F.; BASTOS, M. L. Simultaneous determination of amphetamine derivates in human urine after SPE extraction and HPLC-UV analysis. Biomed. Chromatog., v. 18, n.2, p.125-131, 2004.

TAGLIARO, F.; VALENTINI, R.; MANETTO, G.; CRIVELLENTE, F.; CARLI, G.; MARIGO, M. Hair analysis by using radioimmunoassay, high-performance liquid chromatography and capillary electrophoresis to investigate chronic exposure to heroin, cocaine and/or ecstasy in applicants for driving licence. Forensic Sci. Int., v.107, n.1-3, p.121-128, 2000.

TALWAR, D.; WATSON, I. D.; STEWART, M. J. Routine analysis of amphetamine class drugs as their naphthaquinone derivatives in human urine by highperformance liquid chromatography. J. Chromatog., $B$, v. 735, n.2, p. 229-241, 1999.

WOOD M, LALOUP M, RAMIREZ FERNANDEZ MDEAL M, JENKINS KM, YOUNG MS, RAMAEKERS JG, DE BOECK G, SAMYN N. Quantitative analysis of multiple illicit drugs in preserved oral fluid bysolid-phase extraction and liquid chromatography-tandem mass spectrometry. Forensic Sci. Int., v. 150, n.2-3, p.227-238, 2005.

WOOD M, DE BOECK G, SAMYN N, MORRIS M, COOPER DP, MAES RA, DE BRUIJN EA. Development of a rapid and sensitive method for the quantitation of amphetamines in human plasma and oral fluid by LC-MS-MS. J. Anal. Toxicol., v. 27, n.2, p.7887, 2003.

Recebido para publicação em 15 de dezembro de 2006. Aceito para publicação em 31 de maio de 2007. 